The Israeli Journal of Aquaculture - Bamidgeh, IJA_72.2020.1227989, 17 pages

CCBY-NC-ND-4.0 • https://doi.org/10.46989/001c.21687

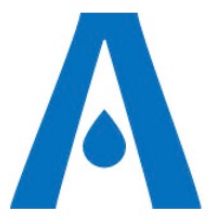

The IJA is a peer-reviewed open-access, electronic journal, freely available without charge to users

Produced by the AquacultureHub non-profit Foundation Sale of IJA papers is strictly forbidden

\title{
The secondary bacterial infection caused by WSSV outbreaks impacts shrimp Marsupenaeus japonicus' growth as well as its intestinal microbiota's composition and function
}

\author{
Zihao $\mathrm{He}^{1+}$, Jichen Zhao ${ }^{1+}$, Xuzheng Liao ${ }^{2}$, Xieyan Chen ${ }^{1}$, Zhibin Fu' ${ }^{1}$, \\ Chengbo Sun ${ }^{13}$ 4* $^{*}$ and Zuotao $\mathrm{Ni}^{5 *}$ \\ ${ }^{1}$ College of Fisheries, Guangdong Ocean University, Zhanjiang, PR China \\ ${ }^{2}$ Southern Marine Science and Engineering Guangdong Laboratory (Zhuhai)/ School of \\ Marine Sciences, Sun Yat-sen University, Guangzhou, PR China \\ ${ }^{3}$ Guangdong Provincial Laboratory of Southern Marine Science and Engineering, \\ Zhanjiang, PR China \\ ${ }^{4}$ Guangdong Provincial Key Laboratory of Pathogenic Biology and Epidemiology for \\ Aquatic Economic Animals, Zhanjiang, PR China \\ ${ }^{5}$ Institute of Oceanology, Chinese Academy of Sciences, Qingdao, PR China
}

Key Words: Marsupenaeus japonicus, WSSV, intestinal microbiota, growth performance, secondary bacterial infections, immunity strategy

\begin{abstract}
Intestinal microbiota homeostasis is a complex ecosystem and is essential for promoting aquatic animals' growth. Invading pathogens can cause dynamical composition and structure change in the hosts' microbiota due to this affecting their functions. WSSV is one of the most common and dangerous shrimp pathogens, which leads to white spot disease with too high mortality. A few studies on shrimp intestinal microbiota mainly focused on the changes during acute infection stage. Marsupenaeus japonicus is one of the most cultured shrimp with a decent ability to cope with environmental changes. In the current research, using $M$. japonicus as a model, we compared the differences of intestinal microbiota between healthy shrimp and the shrimp that survived through the WSSV outbreak. Our study showed that compared to the healthy M. japonicus, the WSSV outbreak disrupted intestinal microbiota structure in the survived shrimp: There was less potential beneficial bacteria and more harmful bacteria. Furthermore, the diversity and total abundance of intestinal microbiota in WSSV-survived shrimp increased significantly. On top of that, a metagenomic analysis by PICRUSt suggested that the changed intestinal microbiota could help the host to combat the secondary bacterial infections caused by WSSV outbreaks by regulating cell growth and death, reducing cell motility, improving energy metabolism, and increasing intestinal enzyme activities regardless the smaller sizes of WSSV-survived shrimp. This could be a result of excessive energy consumption and reduced nutrient-absorbing intestinal microbiota. Our study indicated that shrimp intestinal microbiota plays a fundamental role in combating against secondary infections and regulating the hosts' growth post WSSV outbreaks. The eventual goal is to development of more efficient diagnostics and therapeutic strategies.
\end{abstract}

\footnotetext{
* Corresponding author: Chengbo Sun, suncb@gdou.edu.cn; Zuotao Ni, nizuotao07@qdio.ac.cn; ${ }^{+}$These authors contributed equally to this work
} 


\section{Introduction}

Kuruma shrimp Marsupenaeus japonicus is a popular seafood of great economic value. It is also one of the most cultured shrimps in China. The Kuruma shrimp can adapt to environmental stresses faster and have a high survival rate after long-distance transportation (Zeng et al., 2020). It was reported that in 2018, 55,228 tons of kuruma shrimp were harvested, which brought substantial economic benefits to shrimp farmers. However, with the rapidly increasing prawn production, frequent outbreaks of various diseases followed (Zhang et al., 2020).

When the environmental conditions change suddenly, it often leads to large scales of shrimp infections causing by multiple pathogens, resulting in a high shrimp mortality rate and economical losses. White spot syndrome virus (WSSV) is one of the most dangerous and prevalent viruses of shrimp, which can cause devastating white spot disease with extremely high mortality (Li et al., 2013). There are studies showed that WSSV outbreak contributed to the secondary infection by other pathogens like Vibrio, which increased the cumulative mortality in shrimps (Phuoc et al., 2009).

Secondary bacterial infections are commonly associated with viral infections. Viral infections damage intestine and simultaneously defect innate immune responses that provide a favorable environment for the bacterial growth and facilitate its invasion into healthy sites (Manna et al., 2020). The potential cooperation of bacteria and viruses in promoting disease development has received extensive attention in human recently. There are studies focusing on human showed that, during an infection, viruses may interact substantially and intimately with the commensal microbiota (Xu et al., 2020). The antiviral immune responses induced by acute respiratory infections, such as influenza, are associated with changes in microbial composition and function ("dysbiosis") in the respiratory and gastrointestinal tract. In turn, these changes may alter subsequent immune functions against a secondary bacterial infection or the dynamics of inter-microbial interactions. As a consequence, the proliferation of potential pathogenic bacterial species are enhanced (Xu et al., 2020). There is limited similar research on other animals, including the decapod crustacean.

As a complex ecosystem, the structure and function of shrimp intestinal microbiota are closely related to the physiological process of nutrition metabolism, energy balance, immune defense and gastrointestinal development, which are essential to maintain the stability of internal environment (Zhang et al., 2015). Research that focuses on improving the growth rate and disease resistance in shrimps by maintaining the dynamic balance of intestinal microbiota has become a new hot research area (Munaeni et al., 2020). Pathogen infection often leads to changes in the composition and function of shrimp intestinal microbiota. A previous meta-analysis demonstrated that when a healthy Litopenaeus vannamei was infected with four different pathogens-causing diseases, such as retardation, mysis mold syndrome, white feces syndrome (WFS) and acute hepatopancreatic necrosis disease (AHPND), its intestinal microbiota changed significantly (Yu et al., 2018). In Penaeus monodon, Vibrio exposure caused bacterial dynamics changes in intestines (Wanilada et al., 2016). In Procambarus clarkii, WSSV infection triggered changes in the intestinal flora of crayfish, indicating that WSSV may cause diseases by altered the homeostatic balance of the crayfish intestine (Chen et al., 2020). Two recent reports revealed that an oral WSSV or injected WSSV challenge could alter the bacterial communities in the intestine of $L$. vannamei (Pilotto et al., 2018; Wang et al., 2019). Nevertheless, these studies of the intestinal microbiota changes mainly focused on the acute infection stage in shrimps with obvious symptoms and the shrimps died within a short time, few of them paid attentions to shrimps that survived the disease outbreaks. The survived shrimps are often in the latent infection stage and have no obvious symptoms even though they may be infected by more than one pathogen ( $\mathrm{Li}$ et al., 2016). The intestinal microbiota may contribute to the long-term struggle between the hosts and pathogens. To better understand the functions of shrimp intestinal microbiota in defending secondary bacterial infection, it is necessary to characterize the intestinal microbiota's dynamic compositions in the WSSV survived shrimps and provide information to help predicate shrimp diseases (Xiong et al., 2017). 
In recent years, due to the rapid development of high throughput sequencing technologies, people can thoroughly analyze the composition of the microbial population and the genes in a specific environment through sequencing the 16S rRNA gene. In this study, we collected intestinal and water samples of M. japonicus that survived the WSSV, employed Illumina Hiseq2500 platform to investigate the composition and diversity of the intestinal and water microbiota. We also analyzed the functions of intestinal microbiota. In addition, the growth performances of healthy shrimp and WSSV-survived shrimp were compared. To our knowledge, this is the first comparison study on the composition and function of the intestinal microbiota in healthy and WSSV survived M. japonicus. Our study illuminated immune responses by intestinal microbiota in $M$. japonicus against a secondary bacterial infection caused by WSSV. We provided information to better understand the molecular pathogenesis of viral infection associated with a secondary bacterial infection. The eventual goal is to development of more efficient diagnostics and therapeutic strategies.

\section{Experimental diets and shrimp rearing}

\section{Materials and Methods}

In this study, the M. japonicus were cultured in a shrimp farm in Zhanjiang, Guangdong Province, China. Healthy juvenile shrimp were divided into two groups. The stocking density was 50 individuals $/ \mathrm{m}^{2}$ and the bottom of the each pond was cover with $40 \mathrm{~cm}$ sand. During the rearing period (182 days, June 28th, 2018- December 27th, 2018), the water quality parameters were monitored and recorded daily. The salinity, $\mathrm{pH}$ and temperature of the seawater range from $22.46 \pm 1.76 \mathrm{ppt}, 7.72 \pm 0.54$ and $28.3 \pm 3.5^{\circ} \mathrm{C}$ respectively. The shrimp were fed with commercial shrimp expanded pellets (Tongwei Co., Ltd., China). After rearing for 97 days (October 3th, 2018), one of the groups had an outbreak of WSSV. The shrimp from this group showed obvious symptoms of white spot disease, and the PCR test results was positive for WSSV while negative for IHHNV and DIV1. During the WSSV outbreak (October 3th, 2018- October 14th, 2018), water was changed $90 \%$ once daily, the dead shrimp were removed and the food amount was adjusted accordingly. After rearing for 108 days (October 14th, 2018), no more dead shrimp were found in the WSSV outbreak group, and the survived shrimp had no visible symptoms of white spot disease. The control group had no symptoms during the rearing period. The culture condition were same for both the control group and the WSSV outbreak group. After rearing for 182 days (December 27th, 2018), the shrimp from the control group and the WSSV outbreak group were randomly selected for PCR to confirm WSSV was not presence in the control group. The primers used for WSSV detection were: ie1- $F$ (5-'ATGGCCTTTAATTTCAAGA') and ie1-R (5-'ACAAAGAATCCAGAAATCTC'), which were designed with Primer Premier 5.0 according to the WSSV ie1 gene sequence. The schematic diagram experimental design is shown in Figure 1.

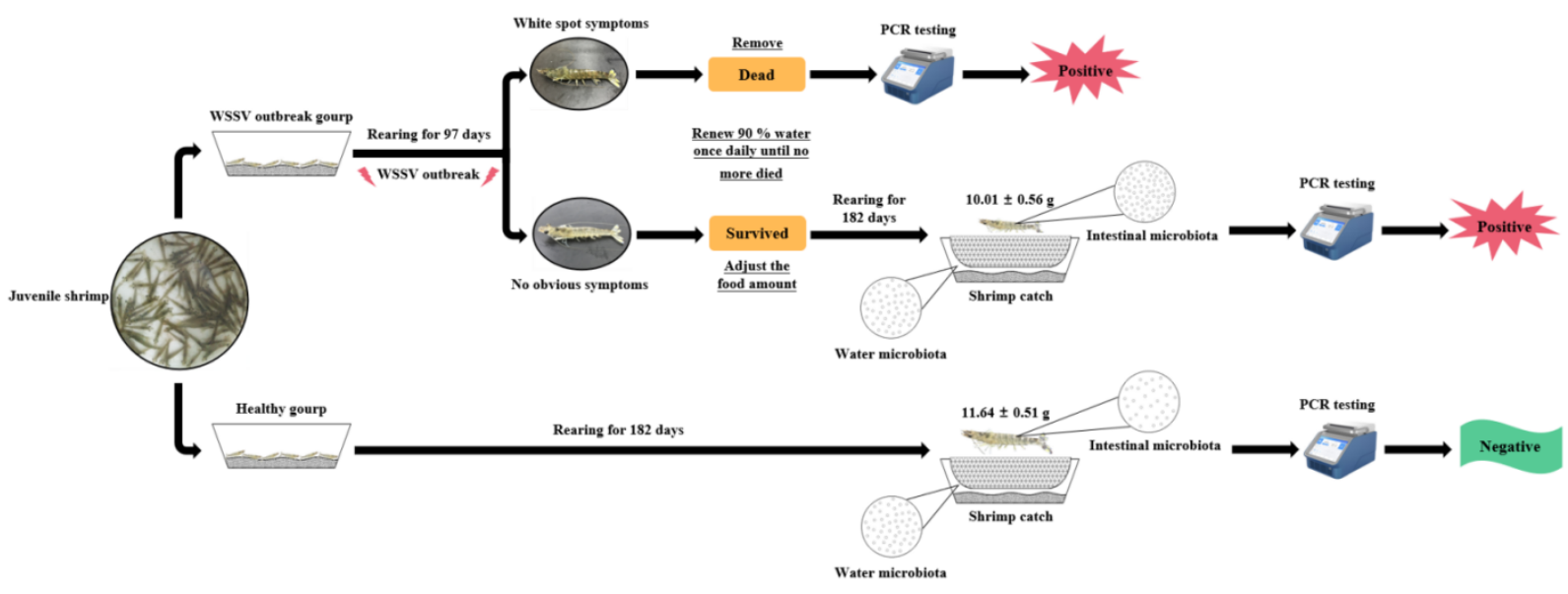

Figure 1 Schematic diagram experimental design. 


\section{Sample collection}

At the end of the rearing period, 30 shrimp were randomly selected from both the control group and WSSV outbreak groups to measure individual body length. Growth rate was calculated by the following formulas:

Weight gain $(W G, \%)=100 \times$ (final body weight - initial body weight) / initial body weight;

Specific growth rate $(S G R, \%)=100 \times($ In final body weight - In initial body weight) / days of the experiment.

Intestine sampling was according to the previously reported methods (Liao et al., 2019). The samples collection was performed under an aseptic condition. 15 extra shrimp were selected randomly from each group, including the WSSV-survived group and the control group. The body surfaces of shrimp were washed with water and $75 \%$ ethanol before dissecting. The intestines were dissected and the intestine of five shrimp from one net cage were mixed in a $1.5 \mathrm{~mL}$ tube on liquid nitrogen, denoted by the labels WSSV-I1, WSSV-I2, WSSV-I3, Control-I1, Control-I2 and Control-I3, and then immediately stored into a $-80{ }^{\circ} \mathrm{C}$ freezer before the DNA extraction. The water sample was collected from 3 representative places $30 \mathrm{~cm}$ below the water surface in each pond with matching places in both the control group and the experiment group. Water samples were filtered through a filter membrane (aperture $0.22 \mu \mathrm{m}$, Shanghai Xingya Purification Material Co., Ltd., Shanghai, China) to collect microbial biomass; 1 filter membrane for each $150 \mathrm{ml}$ water. The biomass from 2 filter membranes for $300 \mathrm{ml}$ water for each sample were combined into a tube and stored at a $-80{ }^{\circ} \mathrm{C}$ freezer before DNA extraction.

\section{DNA extraction and sequencing of $16 S$ rDNA genes}

Total bacterial DNA was extracted by the PowerFecal DNA Isolation Kit (MoBio, Palo Alto, CA, USA) following the manufacturer's directions. The concentration and purity of total DNA were determined by NanoVuePlus Spectrophotometer (GE Healthcare, USA) and $1 \%$ agarose gels. DNA concentration and purity were measured by NanoVuePlus Spectrophotometer (GE Healthcare, USA). The quality of the total DNA was determined by gel electrophoresis in $1 \%$ agarose gels. The primer pair 341F (5-'CCTAYGGGRBGCASCAG-

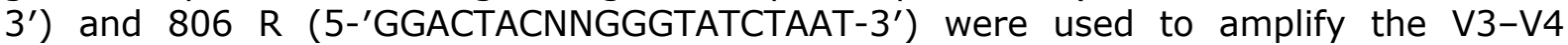
hypervariable region of $16 \mathrm{~S}$ rRNA gene, which was modified with a barcode tag with a random 6-base oligos. Quality of the PCR products were assessed by $2 \%$ agarose gel. Sequencing libraries were generated via using TruSeq DNA PCR-Free Sample Preparation Kit (Illumina, San Diego, CA, USA). The quality of libraries was assessed by Qubit 2.0 Fluorometer (Thermo, USA). The libraries were then subjected to Illumina Hiseq2500 platform sequencing (Guangzhou Sagene Biotech Co., Ltd., Guangzhou, China).

\section{Statistical methods of the intestinal and water microbiota}

Sequences from raw data were analyzed and filtered by Quantitative Insights Into Microbial Ecology (QIIME, http://qiime.org/index.html). Sequences were assigned to the same operational taxonomic units (OTUs) with $97 \%$ similarity by Uparse (Version 7.0.1001, http://drive5.com/uparse/). GreenGene Datebase (http://greengenes.lbl.gov/) was employed to align the sequences. The taxonomic information was annotated by RDP classifier (Version 2.2, http://sourceforge.net/projects/rdp-classifier/) with $80 \%$ confidence threshold. Subsequent through the analysis of alpha diversity and beta diversity to evaluate the abundance and diversity of microbial communities within the samples and the difference in microbial community structure between the samples. The shared and unique OTUs between two groups were Figureure out by Venn diagrams. Alpha diversity index including ACE, Chao1, observed OTUs, Shannon, Simpson, etc. Beta diversity index is based on the phylogenetic relationship between OTUs to calculate the Unifrac distance (weighted Unifrac) and showed the results of Beta diversity through PCoA. In order to further explore the differences in community structure among the grouped samples, statistical analysis methods such as T-test, MetaStat, LEfSe, Anosim, MRPP, and 
MetaGenomeSeq were used to test the significance of the differences in the group composition and community results of the grouped samples to find different species. A Venn diagram was generated to represent the number of unique and shared species among percentages and groups of OTUs.

Functional analysis of the community

The functional characteristics of bacterial communities were predicted by the tool Phylogenetic Investigation of Communities by Reconstruction of Unobserved Species (PICRUSt), employing the 16S rRNA genes as markers using the Greengenes database for taxonomical classification and the Kyoto Encyclopedia of Genes and Genomes (KEGG) annotations for prediction of functional pathways. The Nearest Sequence Classification Index (NSTI) was used to assess the accuracy. The abundance clustering heat map which is standardize by row intuitively showed that the predicted functions of intestinal microbiota in each sample.

\section{Accession number}

Raw data of all samples in this study has been deposited in Sequence Read Archive database of NCBI with the accession number SRP273030.

PCR assay for WSSV

\section{Results}

The PCR amplification results showed that a 675 bp DNA sequence corresponding to WSSV was generated in M. japonicus' intestine samples (Figure 2). The copy number of the WSSV was significant higher in shrimp at WSSV infected acute stage, and lower in WSSV survived shrimp. In contrast, there was no visible copies of the WSSV in the healthy shrimp.

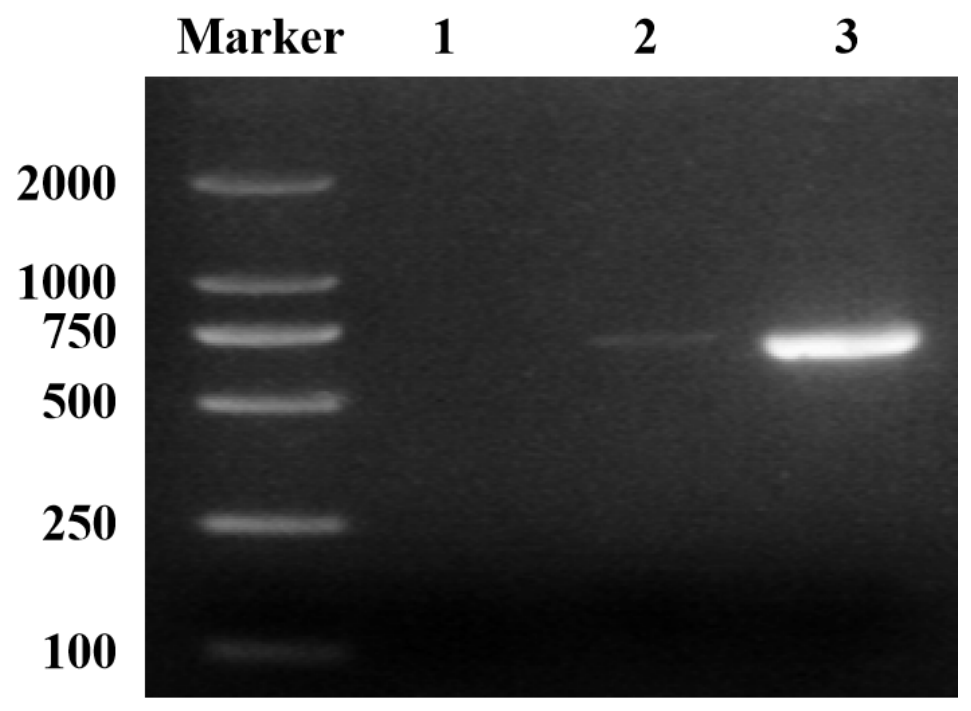

Figure 2 Detection of WSSV with the PCR method. M: DL2000 molecular mass marker; Lane 1: PCR amplified products with DNA template samples extracted from healthy shrimp (control group); Lane 2: PCR amplified products with DNA template samples extracted from WSSV-survived group; Lane 3: PCR amplified products with DNA template samples extracted from WSSV acute infection shrimp. 
Growth performance

WSSV outbreak led to lower growth rate of M. japonicus. As showed in the Table 1, the healthy shrimp had the final weight $(11.64 \pm 0.51 \mathrm{~g})$, final length $(9.63 \pm 0.17 \mathrm{~cm})$, SGR $(2.55 \pm 0.02 \%)$ and WG (10222.39 $\pm 450.38 \%)$, significantly higher than the WSSVsurvived shrimp with the final weight $(10.01 \pm 0.56 \mathrm{~g})$, final length $(9.13 \pm 0.25 \mathrm{~cm})$, SGR $(2.46 \pm 0.03 \%)$ and WG $(8758.54 \pm 493.69 \%)(p<0.01)$.

Table 1 Growth performance of M. japonicus at different health status for 182 days.

\begin{tabular}{cccc}
\hline & \multicolumn{3}{c}{ Growth performance \pm SD } \\
\cline { 2 - 4 } & Control & WSSV & P-values \\
\hline Initial weight $(\mathrm{g})$ & $0.11 \pm 0.02$ & $0.11 \pm 0.02$ & $<0.0001$ \\
Final weight $(\mathrm{g})$ & $11.64 \pm 0.51$ & $10.01 \pm 0.56$ & \\
Initial length $(\mathrm{cm})$ & $2.03 \pm 0.12$ & $2.03 \pm 0.12$ & $<0.0001$ \\
Final length $(\mathrm{cm})$ & $9.63 \pm 0.17$ & $9.13 \pm 0.25$ & $<0.0001$ \\
SGR(\%) & $2.55 \pm 0.02$ & $2.46 \pm 0.03$ & $<0.0001$ \\
WG(\%) & $10222.39 \pm 450.38$ & $8758.54 \pm 493.69$ & \\
\hline
\end{tabular}

Data represent growth performance means \pm SD from three repetitions.

The statistically significant differences between the two groups were calculated by Student's t-test.

Illumina sequencing overview and OTU characterization

A total of 448,392 high-quality sequences were generated from 12 samples, coming from 6 intestinal samples and 6 water samples. The clean reads ranged from 31,941 to 44,414 , with an average of 37,366 reads per sample. The OTU numbers were between 479 and 1260 (Table 2).

Table 2 Summary of OTU numbers and classification at different levels (phylum, class, order, family and genus).

\begin{tabular}{ccccccc}
\hline & \multicolumn{5}{c}{ Summary of OTU numbers and classification at different levels } \\
\cline { 2 - 6 } & OTUs & Phylum & Class & Order & Family & Genus \\
\hline Control-I1 & 547 & 14 & 29 & 47 & 64 & 67 \\
Control-I2 & 667 & 16 & 35 & 55 & 80 & 90 \\
Control-I3 & 479 & 17 & 33 & 48 & 75 & 74 \\
WSSV-I1 & 1079 & 20 & 41 & 70 & 106 & 130 \\
WSSV-I2 & 1260 & 21 & 44 & 81 & 116 & 153 \\
WSSV-I3 & 898 & 18 & 44 & 74 & 108 & 128 \\
Control-W1 & 827 & 24 & 59 & 80 & 94 & 100 \\
Control-W2 & 1189 & 34 & 73 & 97 & 125 & 141 \\
Control-W3 & 1123 & 33 & 73 & 97 & 123 & 132 \\
WSSV-W1 & 755 & 26 & 55 & 74 & 89 & 92 \\
WSSV-W2 & 1004 & 32 & 66 & 88 & 711 & 122 \\
WSSV-W3 & 729 & 26 & 49 & 93 & 97 \\
\hline
\end{tabular}

After the alignment, the sequences of intestine and water samples were clustered into 2684 and 2595 OTUs respectively with a $97 \%$ sequence similarity. Among them, 595 OTUs (22.2\%) from intestine samples were same in both the control group and the WSSV group while 803 OTUs (30.9\%) from water samples were same in the two groups (Figure 3). 


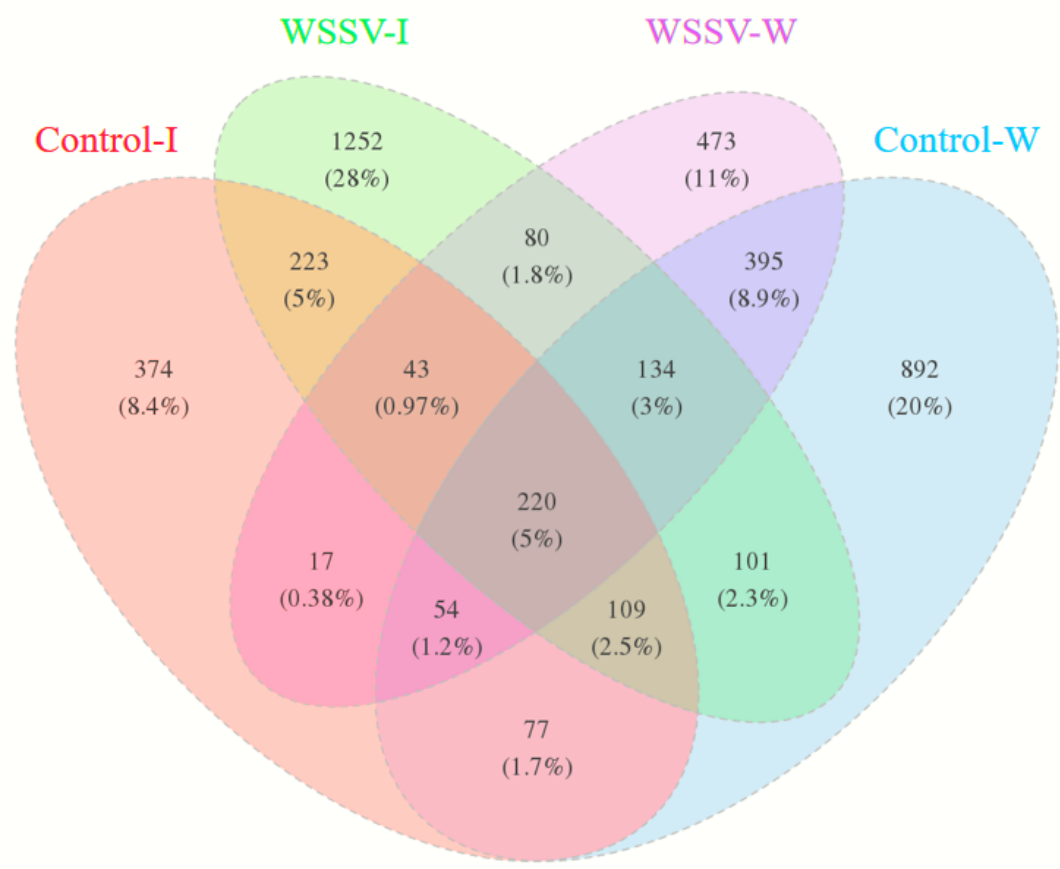

Figure 3 Venn diagram showing the unique and shared OTUs of intestinal and water microbiota in the control group and the WSSV-survived group by using a per os method.

\section{Microbiota differences between control group and WSSV-survived group}

According to the abundance of taxa, OTUs were identified to 48 phyla. In the intestine samples, $89.96 \%$ of the phylotypes from the control group belonged to two dominant phyla: Proteobacteria (75.83\%) and Tenericutes (14.13\%). In the WSSV-survived group, Proteobacteria $(54.26 \%)$ was the most abundant phyla as well, but followed by Cyanobacteria (31.17\%), which the two phyla totally represented $85.42 \%$ of the phylotupes. Within water samples, the dominant phyla were the same. Cyanobacteria $(40.29 \%, 48.99 \%)$ was the most abundant phyla in the control group and the WSSVsurvived group, followed by Proteobacteria $(32.08 \%, 26.08 \%)$ and Bacteroidetes $(18.12 \%$, $18.40 \%$ ), totally accounting for $90.49 \%$ and $93.47 \%$ of the phylotupes, respectively. The other divisions consistently found in the two groups were Actinobacteria, Firmicutes and Verrucomicrobia. (Figure 4A). In the water samples, there was no significant difference of the microbiota between the control group and the WSSV-survived group. In the intestinal samples, most OTUs were mapped to Proteobacteria (Figure 4B), but there was no significant difference between the control group and the experiment group. Additionally, the abundance of Actinobacteria, Cyanobacteria and Firmicutes in the WSSV-survived group was upregulated significantly. In contrast, Tenericutes, the core microbiome in the healthy shrimp except Control-I1, were reduced in the WSSV group. Comparing with the control group, the microbiota in the intestinal samples of the WSSV-survived group was closer to the water samples (Figure 4C). 
A

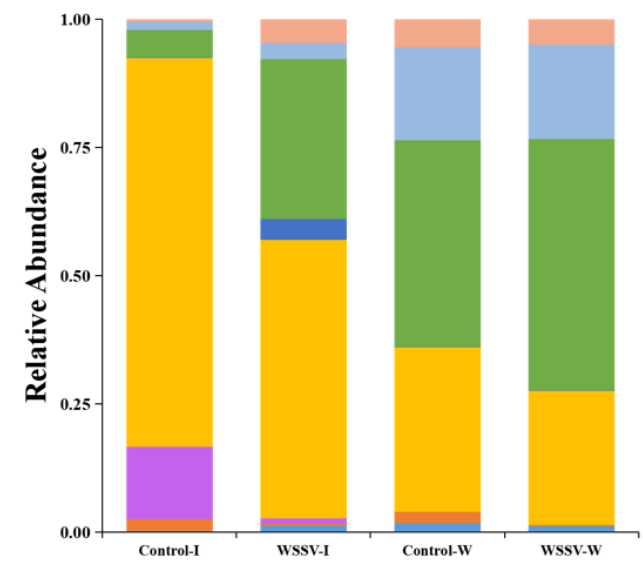

$\mathrm{B}$

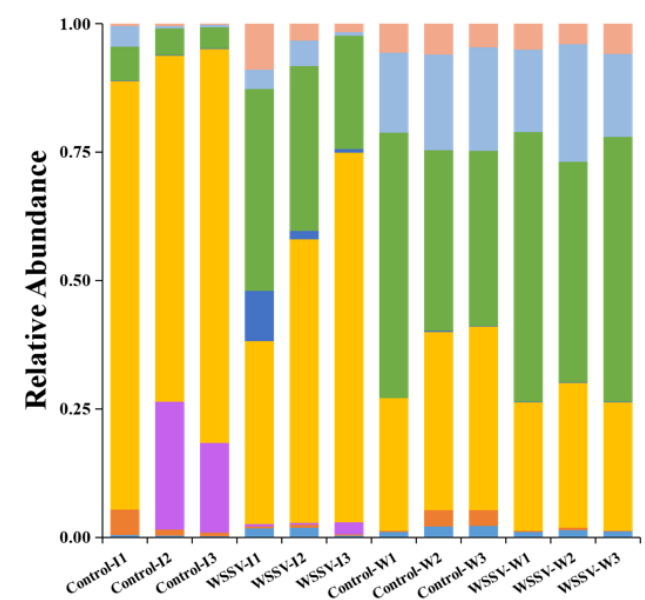

$\mathrm{C}$
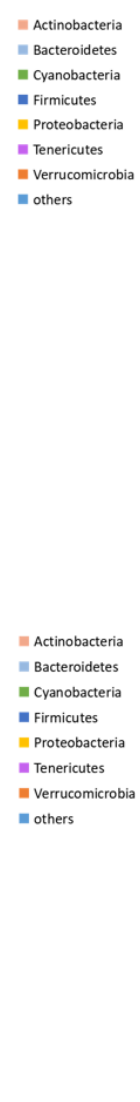
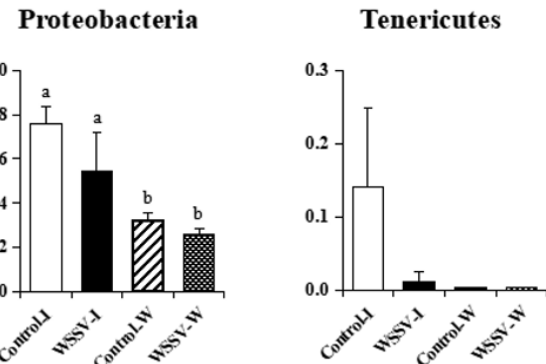

Cyanobacteria

Bacteroidetes

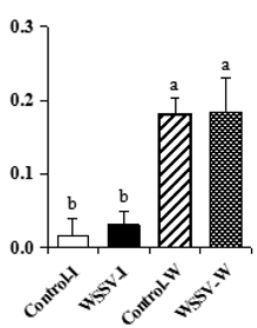

Firmicutes

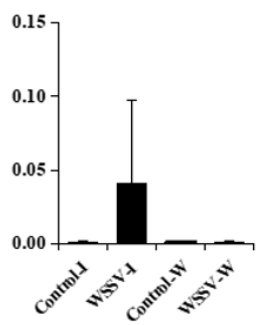

Figure 4 Structure and composition of the intestinal bacterial communities in the control group and the WSSV-survived group on phylum level of taxonomy. (A) means representing as two groups, (B) appearing in each sample and (C) the changes in abundance of dominant bacterial phyla. The data are presented as the mean \pm SD. Dissimilar letters show significant difference and the statistically significant differences between the two groups were calculated by Student's t-test $(P<0.05)$.

At the genus level, a total of 355 taxa were identified. The top 6 genera were Vibrio, Synechococcus, Sphingomonas, Photobacterium, Marivita and Hyphomonas (Figure 5A). Within intestinal samples, the abundance of Synechococcus and Sphingomonas displayed an obvious increase in the WSSV-survived group. In contrast, Photobacterium showed significant decrease in the abundance (Figure 5B). Comparing with the water samples, the abundance of Vibrio in the intestinal samples was higher, while the abundance of Hyphomonas was lower. In addition, the abundance of Synechococcus and Marivita in the intestinal samples of the WSSV-survived group were higher than that of the intestinal samples of the control group, but were similar with the water samples in the two groups (Figure 5C). 
A

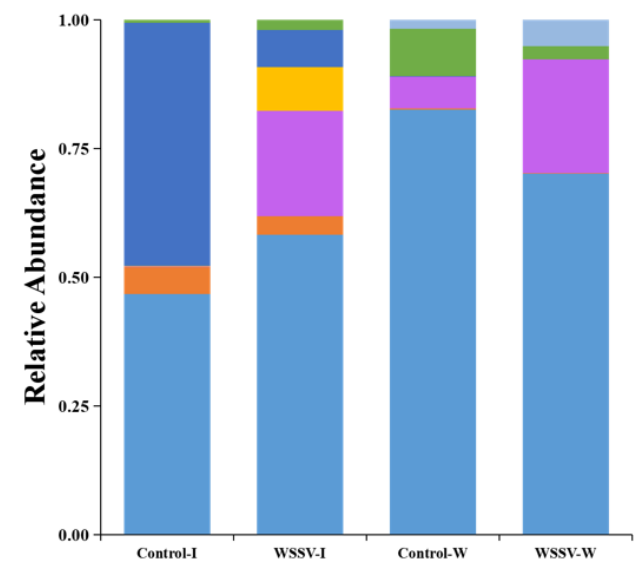

B

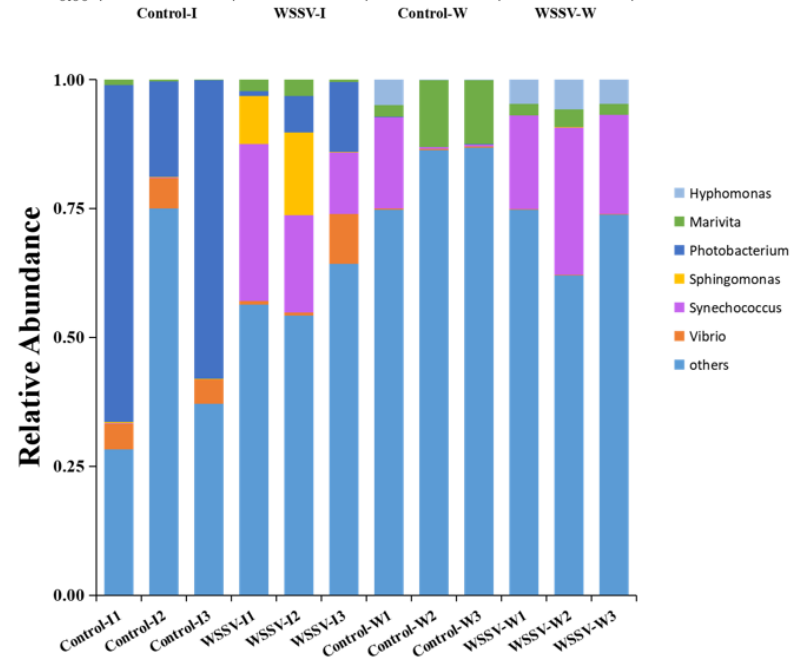

C

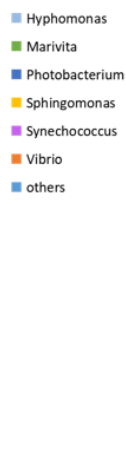

= Marivita

In Sphingomonas

II Synechococcus

V vibrio

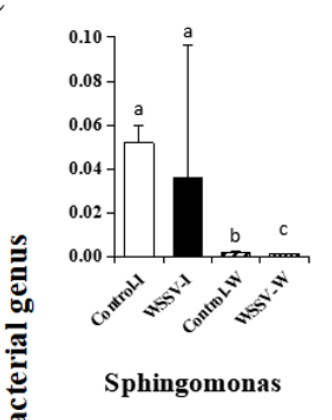

Sphingomonas

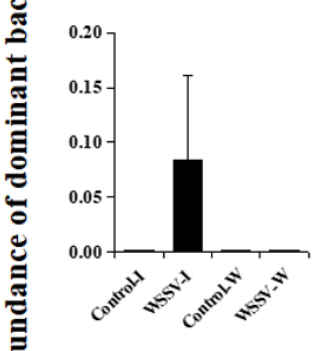

Marivita

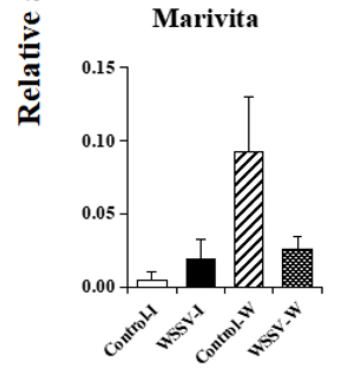

Synechococcus

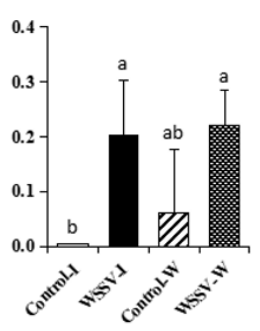

Photobacterium

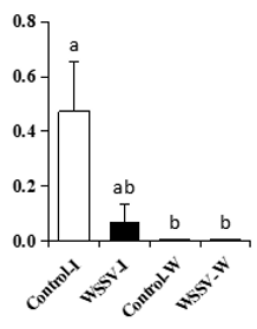

Hyphomonas

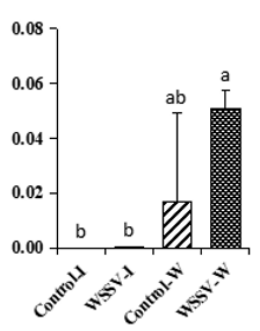

Figure 5 Structure and composition of the intestinal bacterial communities in control group and WSSV-survived group on genus level of taxonomy. (A) means representing as two groups, (B) appearing in each sample and $(C)$ the changes in abundance of dominant bacterial phyla. The data are presented as the mean \pm SD. Dissimilar letters show significant difference and the statistically significant differences between the two groups were calculated by Student's t-test $(P<0.05)$. 
Alpha diversity and beta diversity

Rarefaction curve was often used to estimate the coverage of sequencing. In this study, the rarefaction curves approached the plateau (Figure 6). To investigate the differences of species diversity and richness between two groups, the alpha diversity index was calculated, including observed OTUs, Shannon index, Simpson index, Chao 1 index and ACE index, ranging from 479 to $1260,2.72$ to $6.41,0.64$ to $0.95,779.46$ to 1832.79 and 784.34 to 1892.71 , respectively (Table 3 ). Community richness index (ACE, Chao1 and observed OTUs) and community diversity index (Simpson and Shannon) showed that both of the richness and the diversity were relatively higher in the WSSV-survived group within intestinal samples. However, within water samples, there was no significant difference exist in the diversity and richness between the control group and the WSSV-survived group.

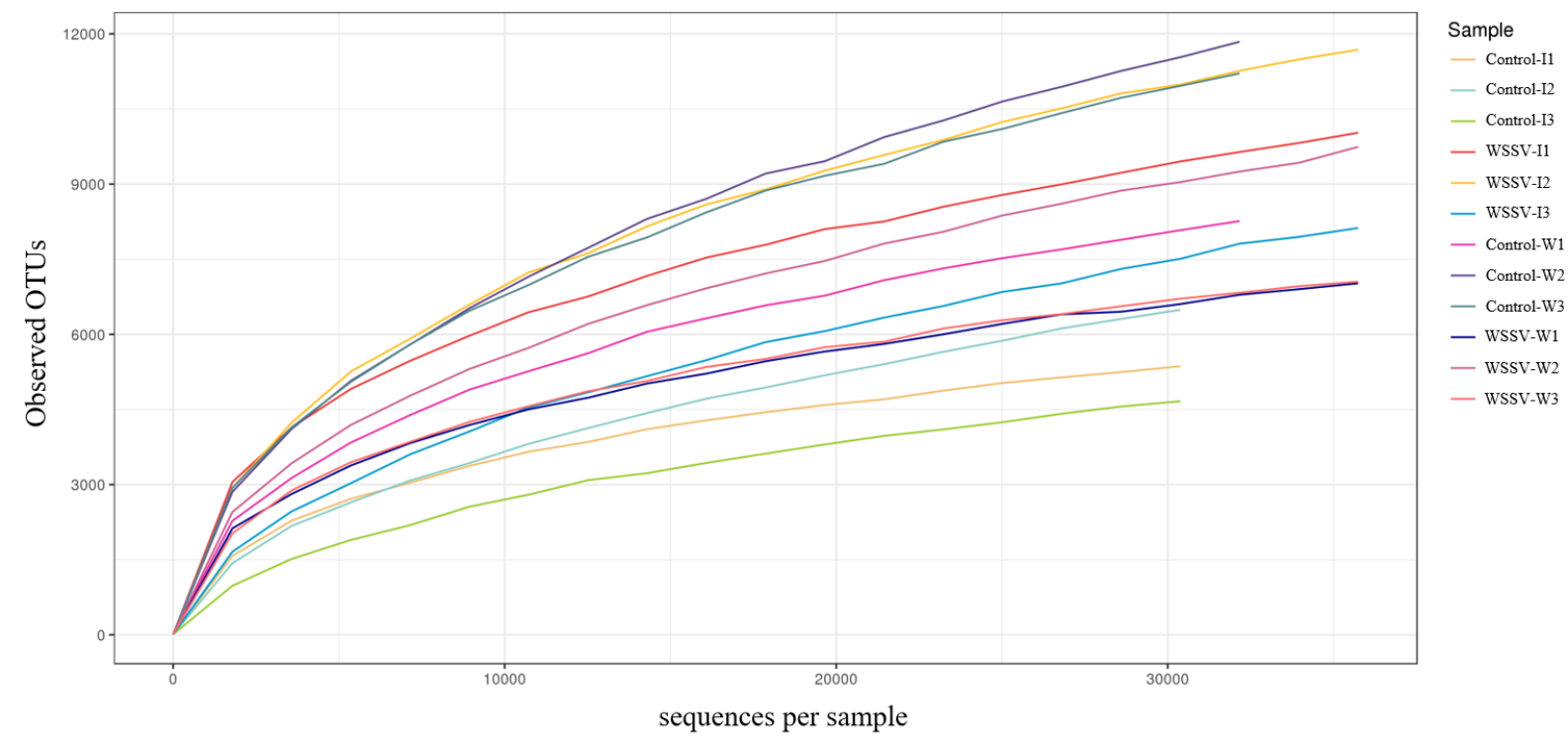

Figure 6 Rarefaction curves of OTUs clustered at 97\% sequence identity across different samples.

Table 3 Richness and diversity indices of intestinal and water microbial in control group and WSSV-survived group.

\begin{tabular}{cccccc}
\hline & \multicolumn{5}{c}{ Richness and diversity indices } \\
\cline { 2 - 5 } & Observed OTUs & Shannon & Simpson & Chao1 & ACE \\
\hline Control-I & $564 \pm 95 \mathrm{a}$ & $3.29 \pm 0.51 \mathrm{a}$ & $0.7 \pm 0.08 \mathrm{a}$ & $938.57 \pm 268.01 \mathrm{a}$ & $962.43 \pm 285.6 \mathrm{a}$ \\
WSSV-I & $1079 \pm 181 \mathrm{~b}$ & $5.65 \pm 1.05 \mathrm{~b}$ & $0.92 \pm 0.04 \mathrm{~b}$ & $1633.9 \pm 131.26 \mathrm{~b}$ & $1639.28 \pm 112.75 \mathrm{~b}$ \\
Control-W & $1046 \pm 192 \mathrm{~b}$ & $5.91 \pm 0.44 \mathrm{~b}$ & $0.93 \pm 0.02 \mathrm{~b}$ & $1573.04 \pm 351.81 \mathrm{ab}$ & $1607.98 \pm 350.37 \mathrm{ab}$ \\
WSSV-W & $829 \pm 151 \mathrm{ab}$ & $5.55 \pm 0.41 \mathrm{~b}$ & $0.92 \pm 0.02 \mathrm{~b}$ & $1279.43 \pm 280.36 \mathrm{ab}$ & $1242.11 \pm 310.6 \mathrm{ab}$ \\
\hline
\end{tabular}

Beta diversity analysis was to comparative analyze the similarity and difference of intestinal and water microbial community in different groups. The PCoA with weighted Unifrac distance showed that all detected samples were divided into three major clusters along PCO1, which could describe $78.75 \%$ of total variation (Figure 7). Indicating that the intestinal microbiome of WSSV-survived shrimp changed significantly compared to healthy shrimp, and the water microbiome was significant difference with the intestinal microbiome though there was no significant difference in the water microbiome between the two groups. 


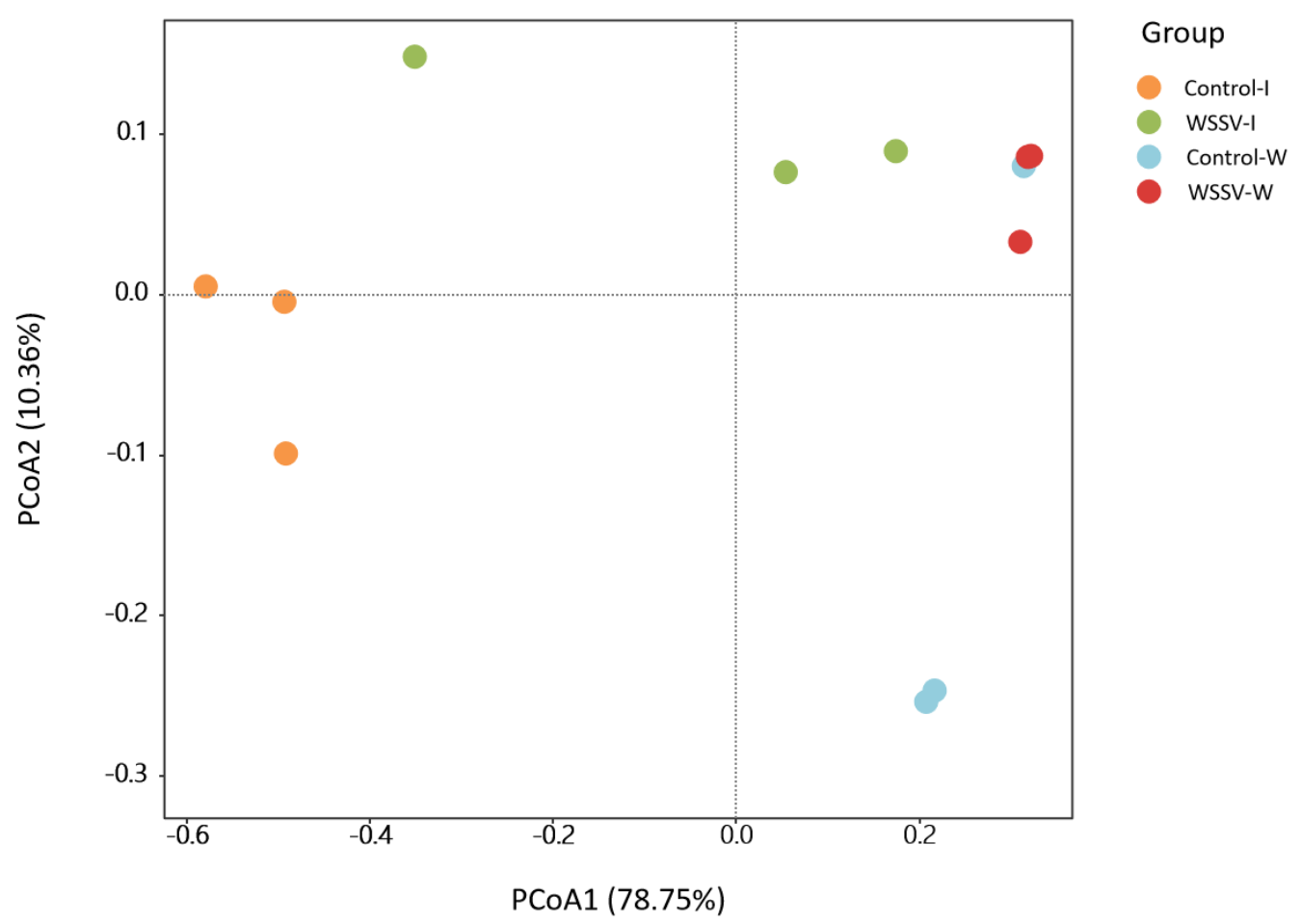

Figure 7 PCoA plot shows the microbial diversity of samples. Samples from the same group were clustered closer.

\section{Functional analysis of the intestinal microbiota}

We used PICRUSt to predict the metagenomic potential between two microbiome groups from shrimp that survived the WSSV outbreak. The accuracy of the prediction was evaluated by NSTI, and the mean number of the samples was $0.073 \pm 0.016$. Results revealed that high abundance of bacterial metagenome in healthy and WSSV-survived shrimp was mainly associated with "Metabolism", "Human Diseases", "Genetic Information Processing", "Environment Information Processing" and "Cellular Processes" in KEGG level 1. In KEGG Level 2, the mean proportion of "Membrane Transport", "Replication and Repair", "Amino Acid Metabolism", "Carbohydrate Metabolism" and "Energy Metabolism" accounted for more than $5 \%$ of the two groups (Figure 8). The abundance clustering heat map which was standardized by row intuitively showed that the predicted functions of intestinal microbiota in each sample. Within "Environmental Information Processing", abundance of "membrane transport" in WSSV-survived group were significantly higher than the control group. Within "Genetic Information Processing", abundance of "Replication and Repair" and "Translation" in WSSV-survived group were significantly higher than those in the control group. Within "Metabolism", abundance of most pathways related to metabolism were increased in the WSSV-survived group, among them, "Amino Acid Metabolism" (10.06\%), "Carbohydrate Metabolism" (9.20\%) and "Energy Metabolism" $(9.46 \%)$ accounted for a large mean proportion in the WSSV-survived group (Figure 9).

The distinct changes on a deeper resolution level, both level 2 and level 3, within "Cellular Process" were showed in Table 4. In KEGG level 2, the mean proportion of "Cell Growth and Death" was exhibited significant increase after the WSSV outbreak $(p<0.05)$, while "Cell Motility" was significant decrease $(p<0.05)$. Within "Transport and Catabolism", the mean proportion of level 3 term "Peroxisome" in the WSSV-survived group $(89.24 \%)$ was significantly higher than the control group $(66.01 \%)(p<0.05)$. In contrast, the mean proportion of level 3 term "Lysosome" in the WSSV-survived group $(10.36 \%)$ was significantly lower than the control group $(33.48 \%)(p<0.05)$. 


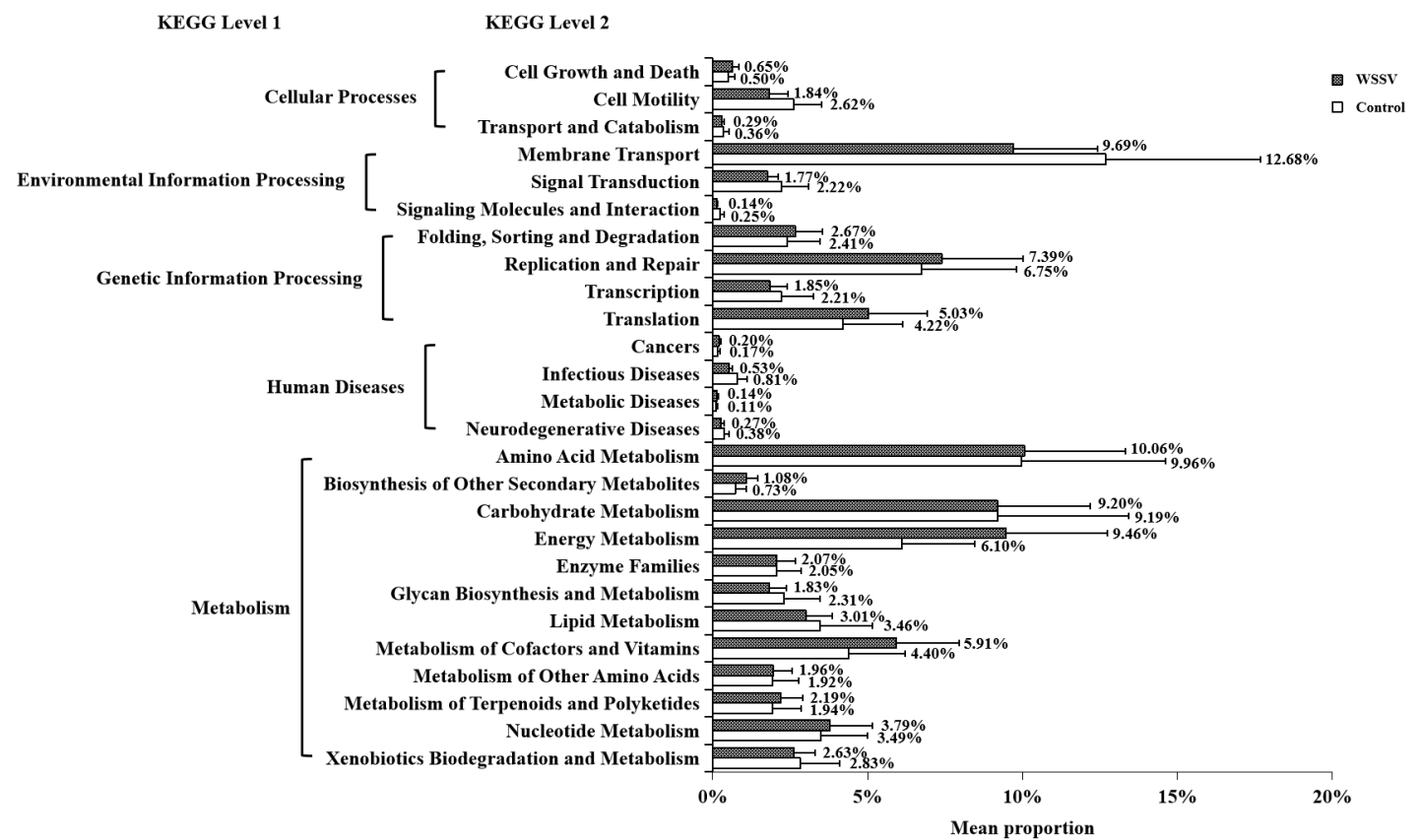

Figure 8 Predicted functions of intestinal microbiota in healthy and WSSV-survived shrimp. The data are presented as the mean \pm SD.

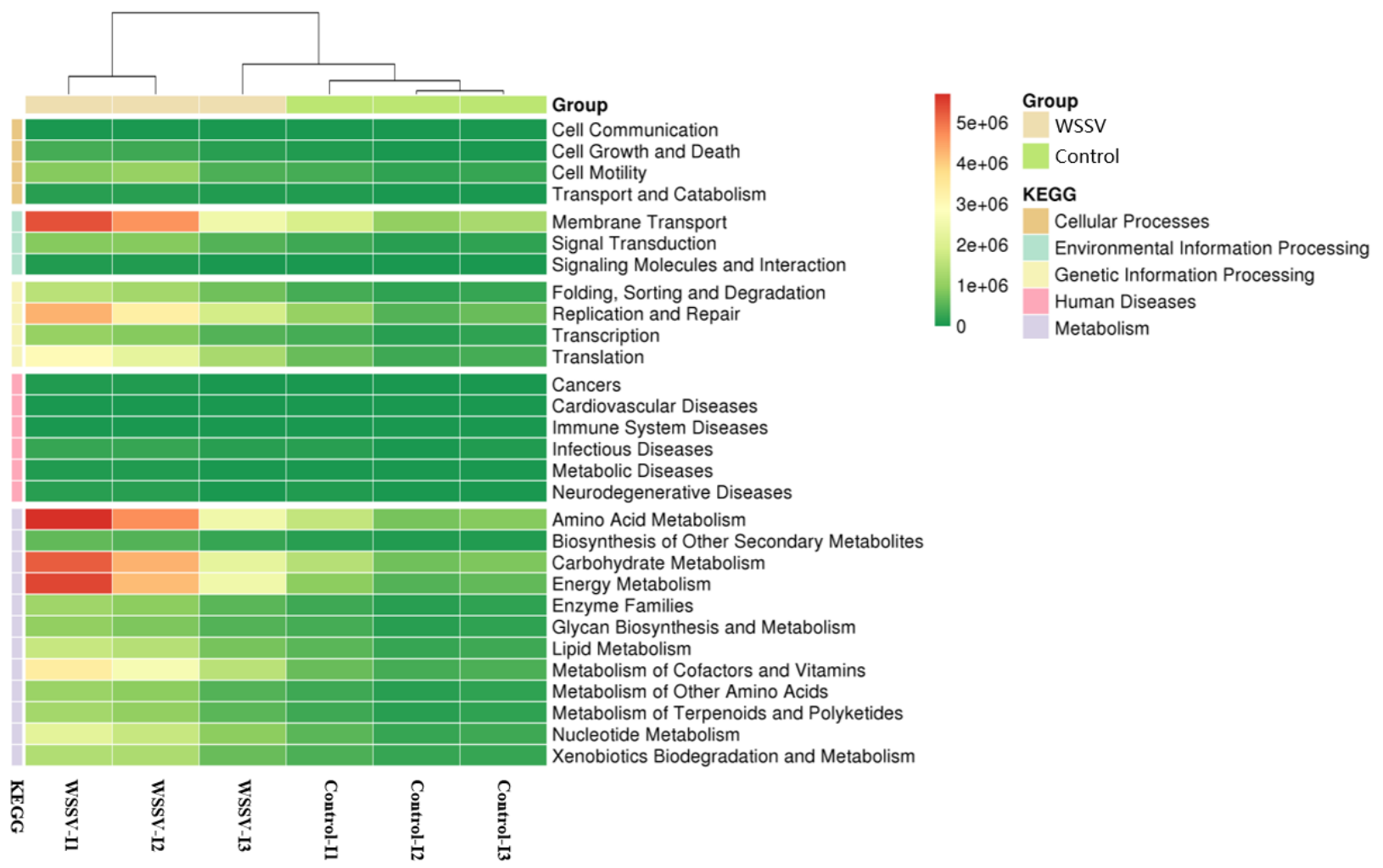

Figure 9 Abundance clustering heat map of predicted functions of intestinal microbiota in each sample (KEGG level 2). Standardize by row. 
Table 4 Relative abundance in KEGG level1, level2 and level 3 within Cellular Process.

\begin{tabular}{|c|c|c|c|}
\hline \multirow[b]{2}{*}{ KEGG level } & \multicolumn{3}{|c|}{ Relative abundance within Cellular Process } \\
\hline & KEGG pathway term & CG (\% of parent) & EG (\% of parent) \\
\hline 1 & Cellular Processes & & \\
\hline 2 & Cell Growth and Death & $14.41 \%$ & $23.26 \%$ \\
\hline 3 & Apoptosis & $3.16 \%$ & $3.11 \%$ \\
\hline 3 & Cell cycle - Caulobacter & $83.39 \%$ & $84.61 \%$ \\
\hline 3 & Meiosis - yeast & $7.72 \%$ & $9.08 \%$ \\
\hline 3 & p53 signaling pathway & $5.73 \%$ & $3.20 \%$ \\
\hline 2 & Cell Motility & $75.29 \%$ & $66.14 \%$ \\
\hline 3 & Bacterial chemotaxis & $18.09 \%$ & $16.74 \%$ \\
\hline 3 & Bacterial motility proteins & $51.60 \%$ & $54.33 \%$ \\
\hline 3 & Cytoskeleton proteins & $10.26 \%$ & $12.53 \%$ \\
\hline 3 & Flagellar assembly & $20.05 \%$ & $16.40 \%$ \\
\hline 2 & Transport and Catabolism & $10.28 \%$ & $10.60 \%$ \\
\hline 3 & Endocytosis & $0.51 \%$ & $0.40 \%$ \\
\hline 3 & Lysosome & $33.48 \%$ & $10.36 \%$ \\
\hline 3 & Peroxisome & $66.01 \%$ & $89.24 \%$ \\
\hline
\end{tabular}

\section{Discussion}

With the development of shrimp farming industry, farming modes of high-density, intensive and large-scale production has become popular. Although it brings high economic benefits, it causes frequent shrimp disease outbreaks (Zhang et al., 2020). WSSV is one of the most destructive viral pathogens of shrimp, and WSSV outbreak can result in a high mortality ( $\mathrm{Li}$ et al., 2013). Most previous research focused on the pathogenesis and immune response of shrimps during acute WSSV infection stage (Xiong et al., 2017), while few studies was on the long-term struggle between the host and the pathogen, especially the changes in the composition and function of the intestinal microbiota. In this study, we used a high throughput sequencing technology to explore the $M$. japonicus intestinal microbiota and water microbiota of a healthy group and a WSSV-survived group. The growth rate of two group were also measured and compared. Our results showed that the intestinal microbial composition of $M$. japonicus changed significantly after a WSSV outbreak, and the intestinal microbiota plays an important role in combating a secondary bacterial infection and regulating the host's growth.

The bacterial diversity was closely associated with the functional stability of intestine microbial in shrimps (Duan et al., 2018). Through the analysis of alpha diversity and beta diversity, we found that the diversity and richness of the intestinal microbiota of WSSVsurvived shrimp increased significantly comparing with the healthy shrimp. Previous studies indicated that in the later stages of the disease outbreaks, the intestinal microbiota diversity in the sick shrimp is higher than that in a healthy one (Zhu et al., 2016). This pattern could be attributed to the compromised selection and colonization resistance on the external invaders in a sick shrimp (Xiong et al., 2017; Zhu et al., 2016). Interestingly, in the water samples, there was no significant difference in the richness and diversity of the water microbiota between the two groups, indicating that the water environment of the two groups was similar, and the water microbiota was not the main factor causing the changes in the intestinal microbiota. Therefore, in this study, the WSSV outbreak was the main element that caused changes in the diversity and richness of intestinal microbiota.

Through an in-depth study of the microbiota composition, we found that the main phyla in the intestinal microbial of M. japonicus were Proteobacteria, Tenericutes and Cyanobacteria, and the secondary phyla were Actinobacteria, Bacteroidetes and Firmicutes. Except for Cyanobacteria, they were also the significant compositions of intestinal microbiome in L. vannamei (Xiong et al., 2015). Comparing with healthy shrimp, 
Actinobacteria and Cyanobacteria in WSSV-survived shrimp increased significantly. At the same time, Tenericutes decreased significantly, but there was no significant difference compared to water microbiota. Additionally, both Venn diagram and PCoA demonstrated that the intestinal microbiota in the WSSV-survived shrimp was closer to water microbiota than that of the healthy shrimp. This finding confirmed previous studies' results that the occurrence of shrimp disease weakens its selection on rearing microbes, resulting in more similar communities between shrimp intestine and rearing water (Zhu et al., 2016).

It is noteworthy that members of Cyanobacteria in the rearing water were quite abundant. Some Cyanobacteria specie have been proved to be potential factors for WSSV outbreaks, the harmful have additive effects upon Cyanobacteria and latent infection of WSSV, enhance the toxic effects and mortality (Cao et al., 2018). At the genus level, Synechococcus was found to be the main component of Cyanobacteria. Previous studies have found that some species can secrete cyanobacterial hemolysins, seriously endangering the safety of aquatic animals (Martins et al., 2007). Interestingly, members of Vibrio were accounted for nearly $5 \%$ of total in both of the two groups. Indeed, they may act as opportunistic agents in the secondary infections or be the actual pathogens . Another interesting finding in our study is that the WSSV outbreak obviously reduced the Photobacterium in the intestinal. Previous studies suggested that supplementing a diet with the living Photobacterium damselae improved $L$. vannamei's non-specific immunity and its resistance to WSSV ( $\mathrm{Li}$ et al., 2011). Additionally, we found a high abundance of Sphingomonas in the intestines of WSSV-survived shrimp. Previous studies proved that Sphingomonas can secrete toxins, which are not only toxic to many animals such as corals (Richardson et al., 1998) and juvenile of Haliotis discus discus (Lin et al., 2006), but also infect humans and cause various inflammations and leukemias (Hu et al., 2007). Overall, our results indicated that WSSV outbreak disrupted the stability of intestinal microbiota of $M$. japonicus and reduced the abundance of potentially beneficial bacteria and increased the abundance of harmful bacteria in the intestine.

Investigating the functional diversity of bacterial communities could help to further understand the regulatory role of intestinal microbiome in the host immune response. In this study, the results of PICRUSt revealed that the gut bacterial of $M$. japonicus related to diverse pathways. The intestinal microbiota plays a significant role in regulating metabolic processes (Shirley et al., 2015). A previous study found that, most KOs related to metabolism decreased in the WSSV-infected shrimp, indicating WSSV may disturb the host energy metabolism during the acute infection period (Wang et al., 2019). However, in this study, most of the pathways related to metabolism in the WSSV-survived groups were not significantly inhibited. On the contrary, the proportion and abundance of energy metabolism in the survived-group was significantly higher than that of the control group. From the data, we assume that unlike the shrimp in the WSSV acute infection stage, WSSVsurvived shrimp may combat with the pathogens by regulating the energy metabolic system. Some recent findings showed that, due to its ample enzymatic potential, intestinal microbiota could regulate shrimp food digestion and nutrient absorption (Sha et al., 2016; Dai et al., 2018). In this study, we found that the WSSV outbreak caused a significant decrease in the growth rate of $M$. japonicus. The mean proportion of membrane transport in the WSSV-survived group was decreased by a PICRUSt analysis. As we know, active transportation is not only an important way of transport in membrane transport, but also the main method for cells to absorb the needed nutrients. Our findings implied that the WSSV outbreak reduced the intestinal microbiota's ability to promote shrimp nutrient absorption. Reduced nutrient accumulation and increased energy consumption may be the reason for the lower growth rate of WSSV-survived shrimp comparing to the healthy shrimp.

By further analyzing "Cellular Process", we found that the metagenomics potential of microbial communities in WSSV-survived group in the term of "Cell Growth and Death" was improved, while the term of "Cell Motility" was less. In KEGG level 3 term, the mean proportion of peroxisome significantly increased while the mean proportion of endocytosis and lysosome decreased. In particular, Catalase (CAT) is a marker enzyme of Peroxisome, which can protect organism tissues from toxic substances, and plays a vital role in a host's 
apoptosis, aging, inflammation and tumor formation. Catalase was considered as an index enzyme for detecting the immune function of crustaceans (Liu et al., 2003). For example, MjCAT participated in the intestinal host-microbe homeostasis by regulating ROS level (Yang et al., 2015). Our data suggested that intestinal microbiota could combat with the following secondary bacterial infections after the WSSV outbreaks by regulating cell growth and death, reducing cell motility, improving energy metabolism, and increasing intestinal enzyme activity.

In conclusion, our study revealed that there are dynamic changes in intestinal microbiota composition and function of WSSV-survived M. japonicus, which structured to help host combat with the secondary bacterial infections caused by WSSV by regulating the growth rate. The proposed model was shown in Figure $\mathbf{1 0 .}$

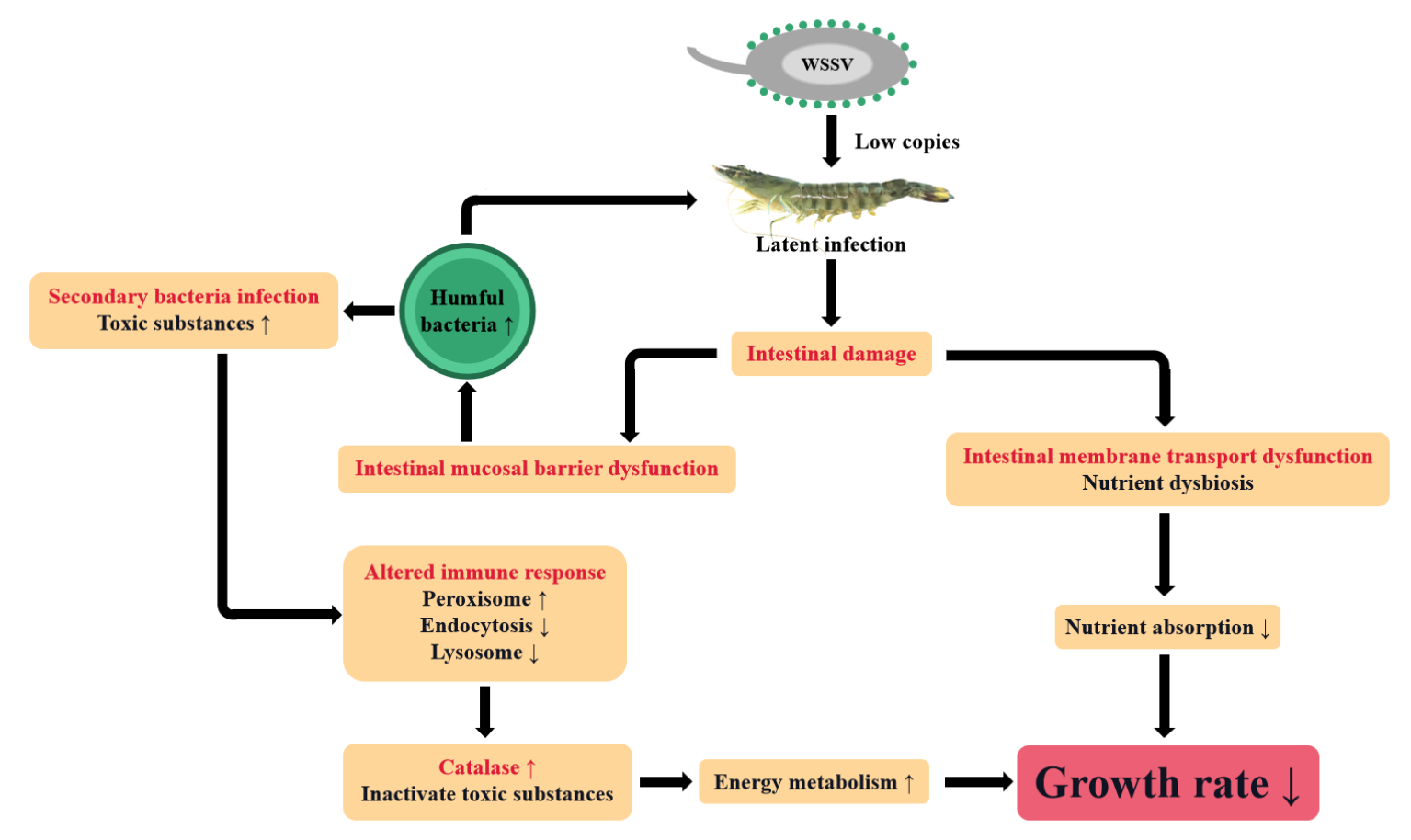

Figure 10 Proposed model for intestinal microbiota defending against secondary bacterial infections.

\section{Acknowledgements}

This research was funded by the key research and development projects in Guangdong Province (Grant No. 2020B0202010009), the project of 2019 Annual Guangdong Provincial Special Financial Fund (Grant No. 2319412525) and the Fangchenggang Science and Technology Plan Project (Grant No. AD19008017).

\section{References}

Cao, Y., Xu, Y., Huang, X., Hu, X., Li, Z., Li, F., Chen, Y., Wen, G., 2018. Lethal effects of microcystis aeruginosa to Litopenaeus vannamei infected by WSSV. Journal of Southern Agriculture 8:1648-1653.

Chen, Y., Gu, Z., Li, L., Chen, T., Yuan, J., 2020. Effects of white spot syndrome virus (WSSV) infection on intestinal flora composition of Procambarus clarkii. Journal of Huazhong Agricultural University 39(2): p. 40 46. 10.13300/j.cnki.hnlkxb.2020.02.006.

Dai, W., Zhang, J., Qiu, Q., Chen, J., Yang, W., Ni, S., Xiong, J., 2018. Starvation stress affects the interplay among shrimp gut microbiota, digestion and immune activities. Fish \& Shellfish Immunology 80:191-199. https://doi.org/10.1016/j.fsi.2018.05.040. 
Duan, Y., Liu, Q., Wang, Y., Zhang, J., Xiong, D., 2018. Impairment of the intestine barrier function in Litopenaeus vannamei exposed to ammonia and nitrite stress. Fish Shellfish Immunol, 78, 279-288. https://doi.org/10.1016/j.fsi.2018.04.050.

Hu, J., He, X., Li, D., Liu, Q., 2007. Progress in research of sphingomonas. Chinese Journal of Applied \& Environmental Biology 13(3):431-437. doi:10.3321/j.issn:1006687X.2007.03.030

Li, G., Song, X., Sun, Y., Mai, K., Xie, G., 2011. Effects of probiotics from the shrimp intestine on the non-specific immunity and antiviral capacity of Litopenaeus vannamei. Journal of Fishery Sciences of China 18(6):1358-1367 doi: 10.3724/SP.J.1118.2011.01358. Li, F., Xiang, J.,2013. Recent advances in researches on the innate immunity of shrimp in China. Developmental \& Comparative Immunology 39(1-2), 11-26. https://doi.org/10.1016/j.dci.2012.03.016

Li, S., Li, F., Sun, Z., Zhang, X., Xiang, J., 2016. Differentially proteomic analysis of the Chinese shrimp at WSSV latent and acute infection stages by iTRAQ approach. Fish \& Shellfish Immunology 54: p. 629-638. https://doi.org/10.1016/j.fsi.2016.05.016.

Liao, X., Hu, S., Wang, B., Qin, H., Zhao, J., He, Z., Chen, X., Liu, Y., Qu, P., Zhang, S., 2019. Dietary supplementation with polypeptides improved growth performance, antibacterial immune and intestinal microbiota structure of Litopenaeus vannamei. Fish \& Shellfish Immunology 92:480-488. https://doi.org/10.1016/j.fsi.2019.06.033.

Lin, X., Huang, J., Zhong, R., Lin, G., Qiu, H., Song, W., 2006. The study on the isolation, identification and drug sensitivity of pathogenic bacterium isolated from juvenile of Haliotis discus discus. Journal of Fuzhou University(Natural Science Edition) 2006(06): 920-924.

Liu, X., 2003. The peroxisome in the tissue cell of penaeus chinensis. Marine Sciences 2003. 27(11): p. 43-46.

Manna, S., Baindara, P., Mandal, S.M., 2020. Molecular pathogenesis of secondary bacterial infection associated to viral infections including SARS-CoV-2. J Infect Public Health 13(10):1397-1404. https://doi.org/10.1016/j.jiph.2020.07.003.

Martins, R., Fernandez, N., Beiras, R., Vasconcelos, V., 2007. Toxicity assessment of crude and partially purified extracts of marine Synechocystis and Synechococcus cyanobacterial strains in marine invertebrates. Toxicon 50(6):791-799. https://doi.org/10.1016/j.toxicon.2007.06.020.

Munaeni, W., Widanarni., Yuhana, M., Wahyudi, A.T., 2020. Impact of dietary supplementation with Eleutherine bulbosa (Mill.) Urb. on intestinal microbiota diversity and growth of white shrimp, Litopenaeus vannamei. 528. https://doi.org/10.1016/j.aquaculture.2020.735466

Phuoc, L., Corteel, M., Thanh, N., Nauwynck, H., Pensaert, M., Alday-Sanz, V., Broeck, W., Bossier, P., 2009. Effect of dose and challenge routes of Vibrio spp. on coinfection with white spot syndrome virus in Penaeus vannamei. Aquaculture 290(1), 6168. https://doi.org/10.1016/j.aquaculture.2009.02.004

Pilotto, M.R., Goncalves, A.N.A., Vieira, F.N., Seifert, W.Q., Bachère, E., Rosa, R.D., Perazzolo, L.M., 2018. Exploring the Impact of the Biofloc Rearing System and an Oral WSSV Challenge on the Intestinal Bacteriome of Litopenaeus vannamei. Microorganisms 6(3), 83. https://doi.org/10.3390/microorganisms6030083.

Richardson, L.L., Goldberg, W.M., Kuta, K.G., Aronson, R.B., Smith, G.W., 1998. Florida's mystery coral-killer identified. Nature, 392(6676):557-558. https://doi.org/10.1038/33302.

Sha, Y., Wang, L., Liu, M., Jiang, K., Wang. B., 2016. Effects of lactic acid bacteria and the corresponding supernatant on the survival, growth performance, immune response and disease resistance of Litopenaeus vannamei. Aquaculture 452:28-36. https://doi.org/10.1016/j.aquaculture.2015.10.014.

Shirley, B., Alejandra, V., Fernanda, C., Karina, R., Samuel, C., Xavier, S., Adrián, O., 2015. Combining metagenomics, metatranscriptomics and viromics to explore novel microbial interactions: towards a systems-level understanding of human microbiome.

Computational and Structural Biotechnology Journal 13:390401.https://doi.org/10.1016/j.csbj.2015.06.001. 
Wang, J., Huang, Y., Xu, K., Zhang, X., Sun, H., Fan, L., Yan, M., 2019. White spot syndrome virus (WSSV) infection impacts intestinal microbiota composition and function in Litopenaeus vannamei. Fish \& Shellfish Immunology 84:130-137. https://doi.org/10.1016/j.fsi.2018.09.076.

Wanilada, R., Amornpan, K., Sawarot, M., Nitsara, K., 2016. Bacterial dynamics in intestines of the black tiger shrimp and the Pacific white shrimp during Vibrio harveyi exposure. Journal of Invertebrate Pathology 133:12-9. https://doi.org/10.1016/j.jip.2015.11.004.

Xiong, J., Wang, K., Wu, J., Qiuqian, L., Yang, K., Qian, Y., Zhang, D., 2015 Changes in intestinal bacterial communities are closely associated with shrimp disease severity. Appl Microbiol Biotechnol 99, 6911-6919. https://doi.org/10.1007/s00253-015-6632-z

Xiong, J., Zhu, J., Dai, W., Dong C., Li, C., 2017. Integrating gut microbiota immaturity and disease-discriminatory taxa to diagnose the initiation and severity of shrimp disease. Environmental Microbiology 19(4), 1490-1501. https://doi.org/10.1111/14622920.13701

Xu, J., Zhao, S., Teng, T., Abdalla, A., Zhu, W., Xie, L., Guo, X., 2020. Systematic Comparison of Two Animal-to-Human Transmitted Human Coronaviruses: SARS-CoV-2 and SARS-CoV. Viruses 12, 244. https://doi.org/10.3390/v12020244

Yang, H., Yang, M., Sun, J., Guo, F., Lan, J., Wang, X., Wang, J., 2015. Catalase eliminates reactive oxygen species and influences the intestinal microbiota of shrimp. Fish \& Shellfish Immunology 47(1):63-73. https://doi.org/10.1016/j.fsi.2015.08.021.

Yu, W., Wu, J., Zhang, J., Yang, W., Chen, J., Xiong, J., 2018. A meta-analysis reveals universal gut bacterial signatures for diagnosing the incidence of shrimp disease, FEMS Microbiology Ecology, 94 (10):147. https://doi.org/10.1093/femsec/fiy147.

Zeng, C., Lin, M., Li, Z., Ma, Y., Wang, S., 2020. The structural and functional characteristics of the gut microbiota of Marsupenaeus japonicus as revealed by $16 \mathrm{~S}$ rRNA gene amplicon sequencing. Microbiology China 47(06), 1857-1866. doi:10.13344/j.microbiol.china.190749

Zhang, J., Duan, Y., Zhang, Z., Dong, H., Li, Z., 2015. Research progress of intestinal microbial flora in shrimp. South China Fisheries Science,11(06):114-119.

Zhang, H., Cheng, W., Zheng, L., Wang, P., Liu, Q., Li, Z., Li, T., Wei, Y., Mao, Y., $\mathbf{Y u}, \mathbf{X} ., 2020$. Identification of a group D anti-lipopolysaccharide factor (ALF) from kuruma prawn (Marsupenaeus japonicus) with antibacterial activity against Vibrio parahaemolyticus. Fish \& Shellfish Immunology 102:368-380. https://doi.org/10.1016/j.fsi.2020.04.039.

Zhu, J., Dai, W., Qiu, Q., Dong, C., Zhang, J., Xiong, J., 2016. Contrasting ecological processes and functional compositions between intestinal bacterial community in healthy and diseased shrimp. Microbial Ecology, 72(4), 975-985. https://doi.org/10.1007/s00248016-0831-8. 